\title{
Plasmon Field Effect Transistor for Plasmon to Electric Conversion and Amplification
}

\author{
Hossein Shokri Kojori ${ }^{\dagger, \|}$, Ju-Hyung Yun ${ }^{\ddagger},{ }^{\dagger}$, Younghun Paik ${ }^{\dagger}$, Joondong $\mathrm{Kim}^{\S}$, Wayne A. \\ Anderson ${ }^{\ddagger}$, Sung Jin Kim ${ }^{\dagger, \#, *}$ \\ ${ }^{\dagger}$ Department of Electrical and Computer Engineering, University of Miami, FL \\ DDepartment of Electrical Engineering, the State University of New York at Buffalo, NY \\ ${ }^{\S}$ Department of Electrical Engineering, Incheon National University, Korea \\ \# Biomedical Nanotechnology Institute (BioNIUM), University of Miami, FL \\ "Both authors contributed equally \\ * Correspondence Author: kim@miami.edu,
}

\section{Device fabrication and characterizations}

Device Fabrication:

First, a heavily doped p-type silicon $\left(<100>\right.$, Boron doping level: $\left.\sim 10^{20} \mathrm{~cm}^{-3}\right)$ substrate was prepared, followed by $100 \mathrm{~nm} \mathrm{SiO}_{2}$ film formation on the $\mathrm{Si}$ wafer using a thermal oxidation for a gate oxide. For the channel layer, a thin n-type $\mathrm{ZnO}$ film (30, 50 and 100nm thickness) was then deposited using an RF magnetron sputtering method. The measured doping concentration of the semiconducting $\mathrm{ZnO}$ film (50nm thick) at $300^{\circ} \mathrm{K}$ is $2 \times 10^{16} / \mathrm{cm}^{3}$ and the electron mobility is $17 \mathrm{~cm}^{2} / \mathrm{v} . \mathrm{s}$ from the Hall effect measurement. The deposition was carried out using a $\mathrm{ZnO}$ target (4 inch size and $99.99 \%$ of purity) applying 300 Watts of RF power under 5 mTorr Ar ambient at $250^{\circ} \mathrm{C}$ of substrate temperature. The deposition rate was modulated to be $7.3 \mathrm{~nm} / \mathrm{min}$, while the substrate was rotated to achieve thickness uniformity. For the channel isolation, the ZnO layer was patterned using a typical photolithography process and a wet etching process using diluted $\mathrm{HCl}$ solution. To create an Ohmic contact between Drain/Source electrodes and the n-type $\mathrm{ZnO}$ film, $\mathrm{Cr} / \mathrm{Au}$ layers were deposited using an electron beam evaporation process. After the lift-off process, the device is a fully functional field effect transistor (FET). The fabricated FET devices were characterized regarding their electrical performance before gold NPs were incorporated onto the active layers. Finally, gold was added on top of the $\mathrm{ZnO}$ channel using a thermal reflow method to create self-assembled gold nanoparticle structures. Several reflow conditions were tested to control the narrow size distribution and to optimize the semi-spherical shape of gold NPs. Best results were obtained with a $5 \mathrm{~nm}$ gold film at $320^{\circ} \mathrm{C}$ heat treatment for 10 minutes. The dimensions of the fabricated devices ranged from $10 \mu \mathrm{m}$ to $100 \mu \mathrm{m}$ of channel length (L) and width (W). The images of the device structure using an optical microscope and a scanning electron microscope (SEM) are shown in Figure 1. It should be noted that the plasmon FET device used for spectral response experiment using 3-MPA (Figure 2b) has an additional $\mathrm{HfO}_{2}$ layer $(10 \AA)$ on top of the $\mathrm{ZnO}$ film for protection during the functionalization process because the weak acidic property of 3-MPA can damage the $\mathrm{ZnO}$ channel. This layer decreases the overall value of spectral response but does not affect the shape of the response curve. 
Device characterization:

For the electrical characterization of the plasmon FET, two source meters (Keithley 2400) are used to provide the Gate-Source and Drain-Source voltage bias. The measured drain current curve shows typical FET characteristics as shown in Figure S1. The spectral response measurement was carried out using a monochromatic light through a fiber-optic cable delivered onto the sample with controlled angles at the probe station. The optical measurement system consists of a quartz tungsten halogen lamp (250W, Oriel), a lock-in amplifier (SR830, Stanford Research) with a mechanical chopper, a monochromator (SP2300, Princeton Instruments) and appropriate optical filters to suppress the second harmonic light from the monochromator. To measure the spectral response of plasmon FET, the lock-in amplifier system was used to calculate the increased drain current. The monochromatic light was modulated $(10-20 \mathrm{~Hz})$ using a mechanical chopper. Since the device has a large DC biased condition, a series resistor was used to measure the voltage, and then the current value was obtained.

The light power was measured using a NIST calibrated silicon photodetector (FDS-1010CAL, Thorlabs) and an optical power meter (PM100D with S120VC, Thorlabs) for the spectral response calculation. The measured monochromatic optical power was controlled from 0.7 to $2 \mathrm{~mW} / \mathrm{cm}^{2}$ for the $400 \mathrm{~nm}-800 \mathrm{~nm}$ spectral range. Additionally, a picoamp meter (Keithley 6485) was used to measure the drain current from the unmodulated (constant) light illumination. A customized LabView program was utilized to control the monochromator, the lock-in amplifier and the picoam meter for the automated computerized measurements.

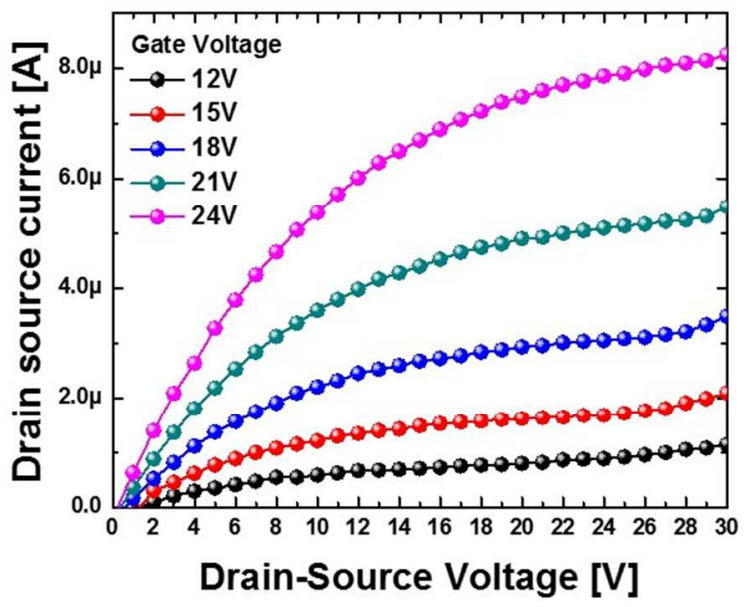

Figure S1. Measured Drain current from the ZnO FET under different voltage bias conditions.

To measure the absorption spectrum of gold NPs, a white light source (Quartz Tungsten Halogen lamp (250W), Oriel) and a spectrometer (StellarNet, BLACK-Comet Concave Grating) were used. For the data in Figure 2, a clean glass substrate (reference sample) and a glass substrate with gold NPs were prepared using the same thermal reflow process condition that was used for plasmon FET fabrication. The samples and light path were maintained 90 degree incident angle. After using a reference sample to measure the baseline of the illumination, the absorption spectrum was measured using the gold NP coated glass substrate. It should be noted that there is 
slightly different plasmonic absorption spectrum between gold NPs on a glass substrate and gold NPs on a plasmon FET due to the refractive index difference and substrate conditions (morphology and thickness). The main purpose of this measurement is to compare the spectral shift before and after 3-MPA coating on gold NPs.

For the data in Figure 3, we prepared a $\mathrm{ZnO}$ deposited glass substrate. Then, gold NPs are incorporated using the same process. We still found a slight difference in absorption spectrum, and we assume that it is due to the different substrate conditions.

\section{Finite Differential Time Domain (FDTD) simulation of plasmonic response of gold nanoparticles:}

To find the relationship between the spectral response and the absorption spectrum of a plasmon FET structure, a FDTD simulation was carried out. The nonlinear dispersion model based FDTD (Rsoft FullWave) was used for this calculation. The gold NPs on top of the ZnO layers are not completely spherical in shape, as shown in Figure S2. The base size of these gold NPs is around $12 \mathrm{~nm}$ which correlates to the average nanoparticle sizes derived from atomic force microscopy (AFM) data. The transmitted power spectrum in the $\mathrm{ZnO}$ layer was obtained under different host matrix conditions for Au NPs (surrounding refractive index, $\mathrm{n}=1,1.5$, and 2). We observed that an increased host material refractive index caused a red shift of the absorption spectrum, as shown in Figure S3. The broader absorption spectrum was obtained with the increased refractive index of the surrounding area.

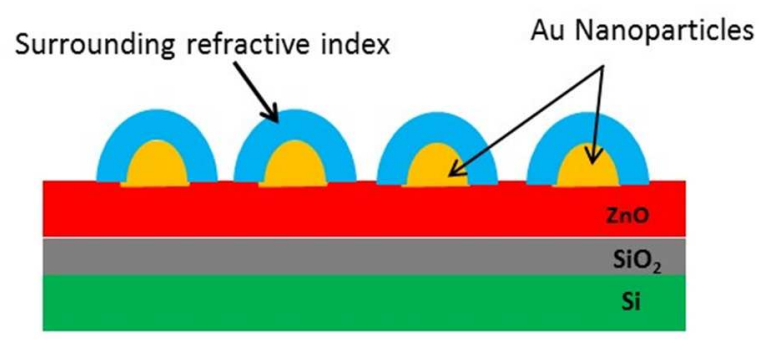

Figure S2. Geometry for FDTD Simulation

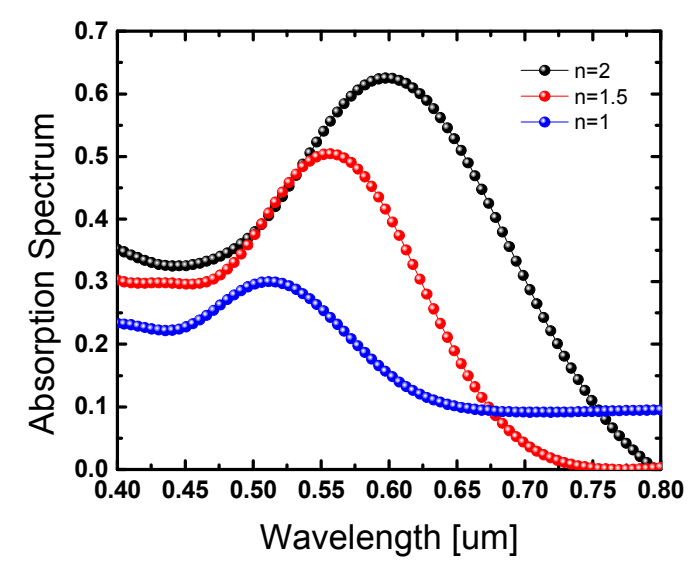

Figure S3. FDTD based simulated results for plasmonic absorbance using the geometry in Fig. $\mathrm{S} 2$ with different gold NP surrounding refractive indices $(1,1.5$ and 2$)$ 


\section{Power and incident angle dependence in the spectral response of the plasmon FET:}

The perfect sphere shape of gold nanoparticles can exhibit a narrow plasmon absorption spectrum, and independency from the polarization and the angle of the incident light. Due to the thermal reflow based fabrication process, the gold nanoparticles are typically formed as a semispherical structure. We carried out an AFM study on the self-assembled gold NPs on various substrates. The AFM image shows hemisphere shaped NP structures which have a larger base size than their height. In order to understand how the hemisphere structure of the NPs changes the spectral response of the plasmon FET, we investigated further by changing the incident angle of the light. Figure S4 shows how incident angle could affect the profile of spectral response of the plasmon FET. The spectral response of the plasmon FET was measured with two different angles, $45^{\circ}$ and $0^{\circ}$ and under well-controlled light intensity conditions, to the plasmon FET. This shows that a $0^{\circ}$ incident angle has a better full-width-half-maximum (FWHM) response and a higher spectral response $(\mathrm{R}=\mathrm{A} / \mathrm{W})$ value than a $45^{\circ}$ incident angle. While the absorption peaks were slightly different in both cases, we believe that the better spectral response from the $0^{\circ}$ incident angle is attributed mainly to the strength of the hot electron momentum inside the gold NPs. It could be explained by using the limited escape cone theory for hot electron emission, which means that the hot electron momentum (kinetic energy) should be greater than the barrier height ${ }^{1}$. Since the initial hot electron momentum is inherited from the incident photon energy (Poynting vector), the incident angle to the gold nanostructure is an important parameter for hot electron emission over the Schottky junction barrier. In addition, we carried out illuminated power dependency studies using neutral density filters which cut $75 \%, 50 \%$, and $25 \%$ of incoming power. As expected, the plasmon detection is a function of the number of photons that produce hot electrons in the gold nanoparticle.

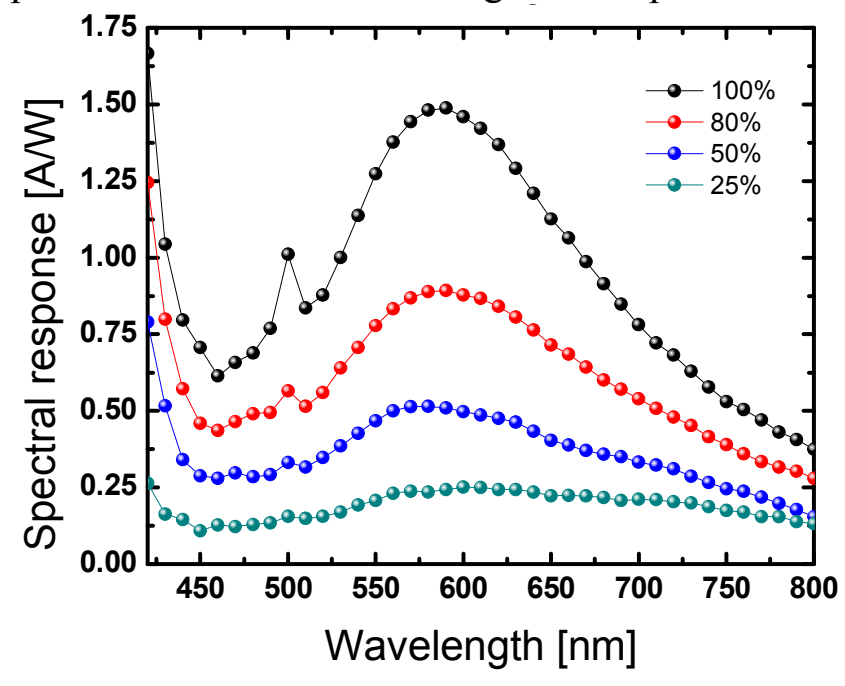

(a)

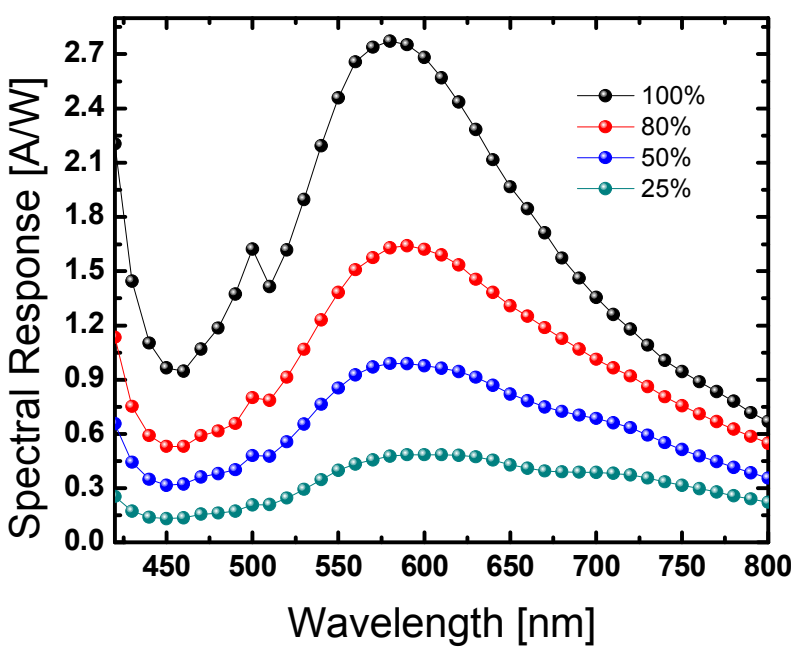

(b)

Figure S4. Spectral response of plasmon FET with different incident power and incident angles (a) $45^{\circ}$, (b) $0^{\circ}$

\section{Plasmon FET under negative gate bias voltage}

Under negative gate bias voltage condition, there is a slight photo response in shorter wavelength. But we did not observe any plasmonic response. There could be two reasons. First, by applying negative gate bias 
for an n-type thin film transistor, the accumulation region could not be formed. Hence, there is no current passing through the semiconductor layer. And the negative bias eventually, blocks the hot electron transfer from metal to semiconductor. The slight increase in shorter wavelength needs further investigation.

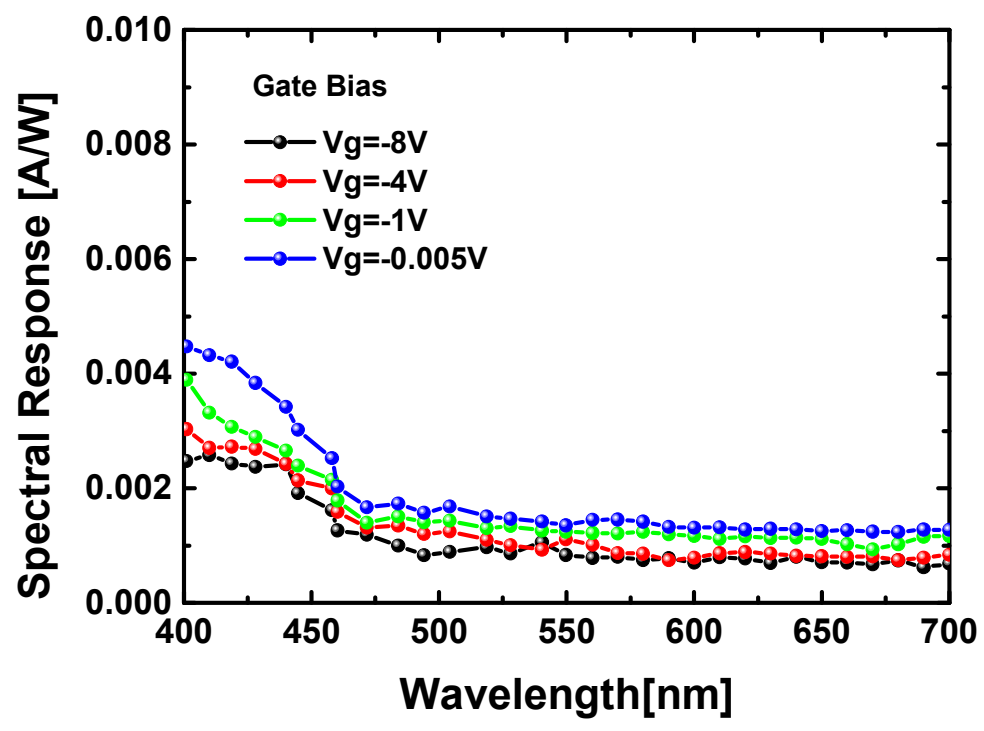

Figure S5. Spectral response of plasmon FET under negative gate bias voltage

\section{Study on free electron discharging}

One of the questions on the working mechanism of the plasmon FET is how the gold nanoparticles can provide hot electrons without depletion when it was illuminated for a long time. If there is a substantial amount of electrons migrated, the gold nanoparticles could be depleted. Consequently, the plasmon FET cannot produce plasmonically induced drain currents, if there is no mechanism to refill the electrons. We carried out an experiment to check if the electrons in the gold nanoparticles can be depleted due to the hot electron migration. We continuously illuminated the plasmon FET and measured the drain current at near the peak wavelength of the plasmonic absorption $\left(0.85 \mathrm{~mW} / \mathrm{cm}^{2}\right.$ at $\left.550 \mathrm{~nm}\right)$. Based on the calculation, the entire volume of gold NPs has total $3 \times 10^{12}$ of free electrons by using the free electron density of gold $\left(5.9 \times 10^{28} / \mathrm{m}^{3}\right)$. Using the spectral response $(0.01 \mathrm{~A} / \mathrm{W})$ without gate voltage bias (no amplification condition, see results in Figure $3 \mathrm{~b}$ ), the number of the collected hot electrons from the gold nanoparticles are $8.9 \times 10^{11} / \mathrm{s}$. Therefore, the estimated time to deplete the entire free electrons from the gold NPs under continuous illumination of light will be $3.37 \mathrm{~s}$. Therefore, several hours of continuous light exposure measurement to observe a depletion of the free electron in gold NPs is more than enough. The measured data in Figure S6 shows that there is no change in plasmonically induced drain current. This indicates that there is a circuit to supply electrons to the gold nanoparticles while it loses plasmonically hot electrons. The sampling rate for data showed in Figure S6 is 10 seconds. After $1 \mathrm{~h}$ dark condition, the light at 550nm was illuminated on sample continuously, and the drain current was stored at every 10 seconds. There have been a report by Liu et al.. ${ }^{2}$ regarding the plasmonically induced hot electron transfer and restoring mechanism. In this paper, they studied an $\mathrm{Au} / \mathrm{ZnO}$ Schottky junction. At the plasmon resonance 
condition, the generated hot electrons in Au NPs overcome the Schottky barrier and leave Au NPs positively charged which brakes equilibrium of Fermi level condition in Schottky junction. As a result, the low energy electrons will flow into Au NPs to compensate the Fermi level differentiation. It should be noted that the gold nanoparticles are not completely isolated from the FET although the nanoparticles are not directly connected to the three electrodes for voltage bias. Therefore, the junction area between $\mathrm{ZnO}$ and gold nanoparticle is under influence from the drain-source voltage bias as shown in Figure S7. The perpendicular electric field at the metal-semiconductor boundary can supply electrons to the gold nanoparticles, while the plasmonically induced hot electrons move to the FET channel (by gate bias) or return to the adjacent gold nanoparticles (by drain bias). This possible mechanism can be supported by the drift current based plasmonic spectral response of plasmon FET under zero Gate voltage bias (Figure $3 b$ in the main text). We also collected the plasmonic induced hot electrons under drain-source voltage bias condition without gate voltage bias. In this case, we have a very low spectral response because the device does not amplify the plasmonic response. But the drain-source voltage bias collects plasmon induced hot electrons without depletion of the free electrons in gold NPs. This means there is a closed circuit between gold NPs and $\mathrm{ZnO}$ channel layer while they have an electric field by drain-source voltage bias.

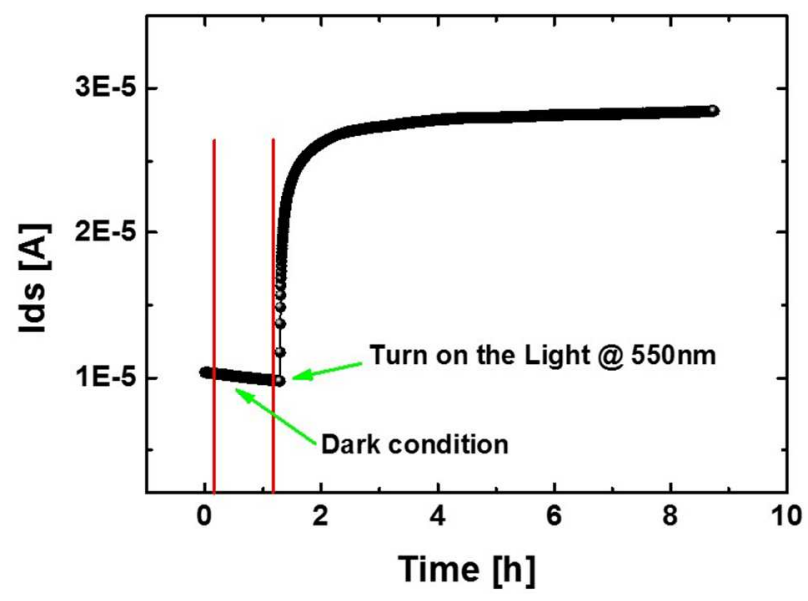


Figure S6. Drain current measurement of plasmon FET under dark and light

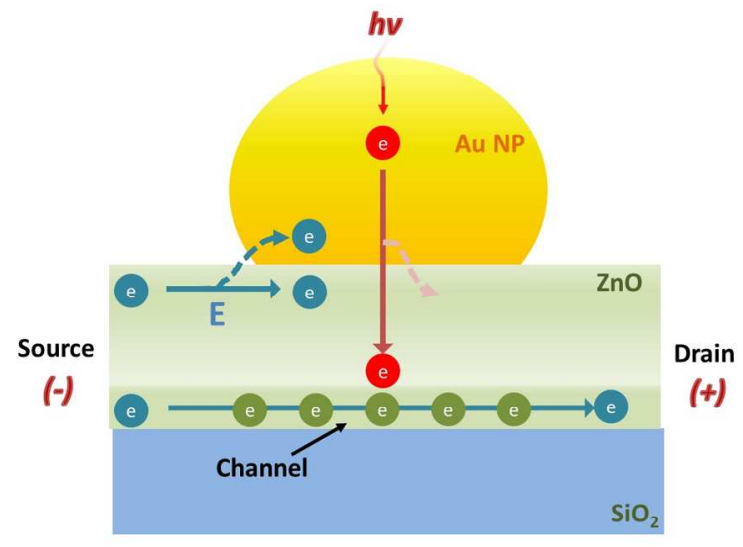

Figure S7. Low energy electrons supply mechanism in plasmon FET

under illumination conditions. The rough $\mathrm{ZnO}$ surface create a complex E-field profile at the boundary and could contribute electron restoration for gold NPs.

\section{Number of contributed hot electrons to the plasmon FET current channel (or internal quantum efficiency) from gold NPs:}

To obtain the number of contributed hot electrons to the plasmon FET current channel form gold NPs, first the total number of photons absorbed by the gold nanoparticles was calculated at the peak wavelength $(\sim 580 \mathrm{~nm}$ or $2.13 \mathrm{eV})$. From the measured absorption spectrum of gold nanoparticles, it has approximately $22 \%$ absorption $(\alpha)$ at the plasmonic peak wavelength. The illuminated power density on the device $(100 \mu \mathrm{m} \times 100 \mu \mathrm{m})$ was $78.5 \mu \mathrm{W} / \mathrm{cm} 2\left(\phi_{i n}\right)$. Therefore, the number of absorbed photons in gold NPs is,

$$
n_{\text {photon }}=\frac{\alpha \cdot \phi_{\text {in }} \cdot A}{q h v}=\frac{0.22 \times\left(78.5 \times 10^{-6}\right) \times\left(10^{-2}\right)^{2}}{\left(1.6 \times 10^{-19}\right) \times 2.13}=5.05 \times 10^{9} / \mathrm{s}
$$

Then, the number of hot electrons that contributed to increased drain current was calculated. Since the transistor is operating under saturation mode, the FET drain current at saturation mode can be written $\mathrm{as}^{3}$ :

$$
\Delta I_{d s}=\frac{1}{2} \mu_{n} C_{o x} \frac{W}{L}\left(V_{g s}-V_{t h}\right)^{2}
$$

Where $\mathrm{C}_{\mathrm{ox}}$ is capacitance per unit area $\left(\frac{\Delta Q}{V \cdot C m^{2}}\right), \mu_{n}$ is the mobility of electrons in $\mathrm{ZnO}$ layer $\left.\left(\frac{C m^{2}}{V . s}\right)\right), V_{g s}$ is the gate-source bias voltage and $V_{t h}$ is the threshold voltage of FET. W and L represents the width and length of channel area in FET structure respectively. 
Since the plasmon FET was under a large voltage bias condition $\left(\mathrm{V}_{\mathrm{ds}}=20 \mathrm{~V}\right.$ and $\left.\mathrm{V}_{\mathrm{gs}}=20 \mathrm{~V}\right)$, the small threshold voltage is neglected. Using the charge is the product of capacitance and voltage $(Q=C . V)$, The increased drain current equation becomes:

$$
\Delta I_{d s}=\frac{1}{2} \mu_{n} \Delta Q \frac{W}{L} * V_{g s}
$$

Experimentally obtained electron mobility $\left(\mu_{n}\right)$ of ZnO TFT was $17 \mathrm{~V} . \mathrm{s} / \mathrm{cm}^{2}$. And the measured drain current change $\left(\Delta I_{d s}\right)$ using lock-in amplifier was $23.4 \mathrm{nA}$ at $580 \mathrm{~nm}$.

Therefore, the increased charge $(\Delta Q)$ can be calculated as follow:

$$
\Delta \mathrm{Q}=\frac{2 \times \Delta \mathrm{I}_{\mathrm{ds}}}{\mu_{\mathrm{n}} \times \mathrm{V}_{\mathrm{gs}}}=\frac{2 \times 23.4 \times 10^{-9}}{17 \times 20}=1.376 \times 10^{-10}[C]
$$

Then the total increased number of electrons at the channel is:

$$
n_{\text {channel_electron }}=\frac{\Delta Q}{q}=\frac{1.376 \times 10^{-10}}{1.6 \times 10^{-19}}=8.6 \times 10^{8}
$$

As a result, around $17 \%\left(8.6 \times 10^{8}\right)$ of total generated hot electrons $\left(5.05 \times 10^{9}\right)$ are contributed to the increased drain current $\left(\Delta I_{d s}\right)$. And this number is defined as quantum efficiency (Q.E.).

As described in the main text, the gate voltage bias facilitates the hot electron transport to the FET channel. Therefore, the plasmon drain current was increased when higher gate voltage bias. Using the same method, we also calculated hot electron contribution efficiency (or QE) under different gate bias conditions. as shown in Figure S8. This shows a unique property of plasmon FET, which is the internal efficiency of photo-generated hot electron efficiency, can be controlled by the gate voltage bias.

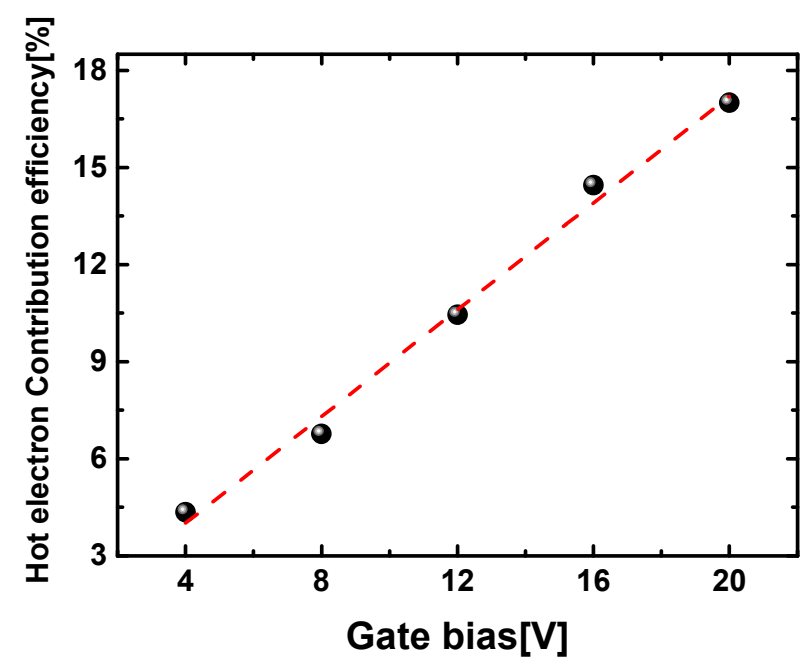


Figure S8. Hot electron contribution efficiency (or IQE) under different gate bias conditions. This set of data was calculated by using the results in Figure 3a. This efficiency is the ratio of contributed hot electrons vs. generated hot electrons.

Finally, the number of hot electrons that each gold NPs contributes to the current channel of plasmon FET can be calculated. In this way, the covered area on plasmon FET is $100 \mu \mathrm{m}$ $\mathrm{x} 100 \mu \mathrm{m}$, and $5 \mathrm{~nm}$ of gold was deposited before the thermal reflow process. Assuming average diameter of $10 \mathrm{~nm}$ for $\mathrm{Au}$ nanoparticles after self-assembly process. Each nanoparticle has a volume of $4.186 \mathrm{x} 10^{-24} \mathrm{~m}^{3}$. The total volume of $\mathrm{Au}$ nanoparticles based on the fabrication condition is $10^{-12} \mathrm{~m}^{3}$. Therefore, the plasmon FET has $1.19 \times 10^{7} \mathrm{Au}$ NPs. Therefore, the number of contributing electrons from each Au NP is $8.6 \times 10^{8} / 1.19 \times 10^{7}=72$.

In addition, we also calculated the quantum efficiency under no gate bias condition. As shown in Figure $3 \mathrm{~b}$, the responsivity becomes extremely small without gate voltage bias. In this case, the device operates as a photoconductor. Thus the drain current can be obtained using following equation:

$$
\Delta I_{D}=q \cdot \mu \cdot \Delta n \cdot E
$$

Based on the measured data shown in Figure $3 b$, the calculated $\Delta \mathrm{n}$ is $1.225 \times 10^{4}$ under $30 \mathrm{~V} \mathrm{~V}_{\mathrm{DS}}$ bias. And it is around $0.0017 \%$ of the generated hot electrons in gold NPs.

\section{Atomic force microscopy (AFM) study}

AFM was carried out to study and visualize the gold NPs distribution on top of the plasmon FET, as shown in Figure S9. The NP shapes are not ideal spherical structures, and the size distributions vary by different substrate conditions. This explains the broad spectral response of the plasmon FETs.

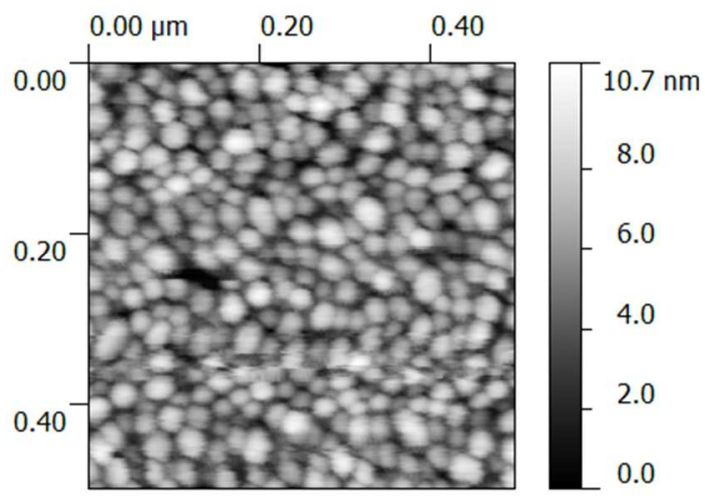

Figure S9. AFM image of gold NPs on top of FET channel

\section{Spectral response from the devices with different $\mathrm{ZnO}$ channel thicknesses}

The plasmon FETs were fabricated with different $\mathrm{ZnO}$ channel thicknesses $(30,50,100 \mathrm{~nm})$ and the spectral responses from each device were characterized. Under the same voltage bias condition, the highest spectral response was observed with the thinnest $\mathrm{ZnO}$ film, as illustrated in Figure S10. This phenomenon can be explained by the distance that the induced hot electrons have to travel to reach the FET current channel. With a longer distance, the chance of 
recombination of hot electrons inside the $\mathrm{ZnO}$ layer also becomes higher. Based on Figure S10, the plasmon FET with $\mathrm{ZnO}$ thickness of $100 \mathrm{~nm}$ almost shows no plasmon spectral response. Also, the plasmon FET with $30 \mathrm{~nm}$ of $\mathrm{ZnO}$ shows the highest Plasmonic signal in comparison to the 50 and $100 \mathrm{~nm}$ thickness $\mathrm{ZnO}$ films. It should be noted that we have achieved highest spectral response with thinner $\mathrm{ZnO}$ channel. But we report the best spectral response from $50 \mathrm{~nm}$ thick $\mathrm{ZnO}$ channel in the main manuscript because the FETs with 50nm channel thickness have better reliability, regarding intrinsic FET operation.

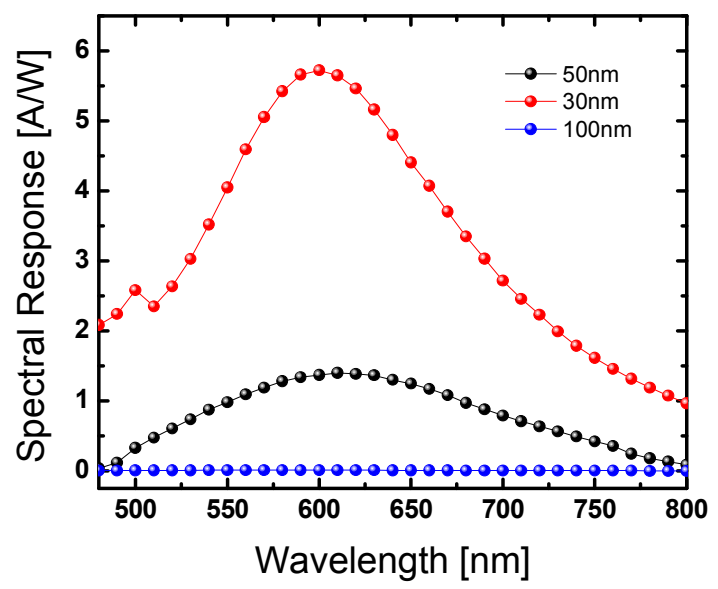

Figure S10. Spectral response of plasmon FET with different thicknesses of ZnO layer (30, 50, $100 \mathrm{~nm})$

\section{Schottky Barrier height for $\mathrm{ZnO} / \mathrm{Au}$ structure}

We carried out an empirical study to find out the Schottky barrier energy between gold and $\mathrm{ZnO}$ film under various conditions. It should be noted that there may be a slight difference between the studied values and the Schottky barrier of the plasmon FET because plasmon FET uses a nanostructured gold for the metal electrode. Schottky diodes of $\mathrm{Au} / \mathrm{ZnO}$ and $\mathrm{Au} / \mathrm{HfO}_{2} / \mathrm{ZnO}$ were fabricated to characterize the Schottky barrier height and the results are shown in Figure S11. The device structure is $\mathrm{Au}(10 \mathrm{~nm}) / \mathrm{HfO}_{2}$ $(0,1,2,3,4 \mathrm{~nm}) / \mathrm{ZnO}(800 \mathrm{~nm}) / \mathrm{AZO}(250 \mathrm{~nm}) / \mathrm{Al}(100 \mathrm{~nm})$ with $1 \mathrm{~mm}$ diameter of circular pattern. For diode fabrication, $100 \mathrm{~nm}$-thick $\mathrm{Al}$ was deposited on a Si substrate. Then, AZO and $\mathrm{ZnO}$ were deposited on the Al layer by a sequence, forming Ohmic and Active layers, respectively. $\mathrm{AZO}$ between $\mathrm{Al}$ and $\mathrm{ZnO}$ prohibits $\mathrm{Al}$ diffusion and provides an Ohmic property. $\mathrm{RF}$ magnetron sputtering was used to deposit the $\mathrm{ZnO}$ and $\mathrm{AZO}$ films using a pure $\mathrm{ZnO}$ target (99.99\%) and $\mathrm{AZO}$ target (99.99\% and $2 \%$ of $\mathrm{Al}_{2} \mathrm{O}_{3}$ sintered). $\mathrm{AZO}$ and $\mathrm{ZnO}$ deposition were carried out with a single pump-down with $250{ }^{\circ} \mathrm{C}$ of heating, and $300 \mathrm{~W}$ of power. Patterning of the diode structure was done by photolithography. An ultra-thin $\mathrm{HfO}_{2}$ layer was deposited using

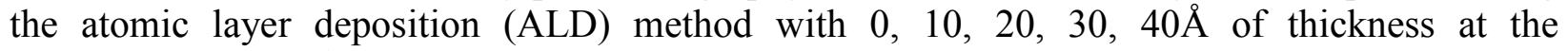
temperature of $120^{\circ} \mathrm{C}$. The gold deposition was followed by thermal evaporation, forming the Schottky junction. After performing lift-off, the diode was isolated by a $\mathrm{HCl}$ wet etching process by removing a portion of the $\mathrm{ZnO}$ and $\mathrm{AZO}$ to reach the back $\mathrm{Al}$ contact. Diode characteristics of ideality factor, dark saturation current and barrier height were extracted based on the ideal thermionic emission equation of Schottky contact in Equation S2-3 and tabled in Table S1. In fact 


$$
J=J_{0}\left(\exp \left(\frac{\mathrm{qV}_{\mathrm{a}}}{n \mathrm{k}_{\mathrm{B}} T}\right)-1\right)
$$

and

$$
J_{0}=A^{* *} T^{2} \exp \left(-\frac{q \phi_{b}}{k_{B} T}\right)
$$

where $A^{* *}$ is the effective Richardson constant for the semiconductor (for $\mathrm{ZnO}, A^{* *}=$ $\left.32 \frac{A}{c^{2} K^{2}}\right)^{4}$, T is the absolute temperature (300K), $q$ is charge of a single electron, $n$ is the ideality factor, $k_{B}$ is the Boltzmann constant, and $V_{a}$ is the applied voltage.

Gold has a high work function with the range of around 5.1-5.47 $\mathrm{eV}^{5}$, which is suitable for Schottky junction formation with the n-type $\mathrm{ZnO}$. Furthermore, ultra-thin $\mathrm{HfO}_{2}$ was introduced to study the thickness dependency on the diode current and tunneling probability. Figure S11 presents semi-log I-V characteristics, which shows current rectification by $\mathrm{Au} / \mathrm{ZnO}$ Schottky barrier. Inserting an $\mathrm{HfO}_{2}$ layer with $10 \AA$ of thickness decreased the current density of both the forward and reverse bias regions while showing better current rectification due to surface passivation effect. Quantum tunneling phenomena enable diode current flow with an insulating layer.

However, insertion of $\mathrm{HfO}_{2}$ layer thicker than 20A, the reverse current was drastically suppressed due to a decrease of tunneling probability. With $40 \AA$ of $\mathrm{HfO}_{2}$ thickness, either forward current was severely limited. This insulated $\mathrm{Au} / \mathrm{ZnO}$ junction possesses a large ideality factor since abnormal conduction mechanism (current blocking by low tunneling probability). These results provide a strong clue about the current flow mechanism in the $\mathrm{Au} / \mathrm{HfO}_{2} / \mathrm{ZnO}$ channel region of the plasmon FET as described in the main manuscript.

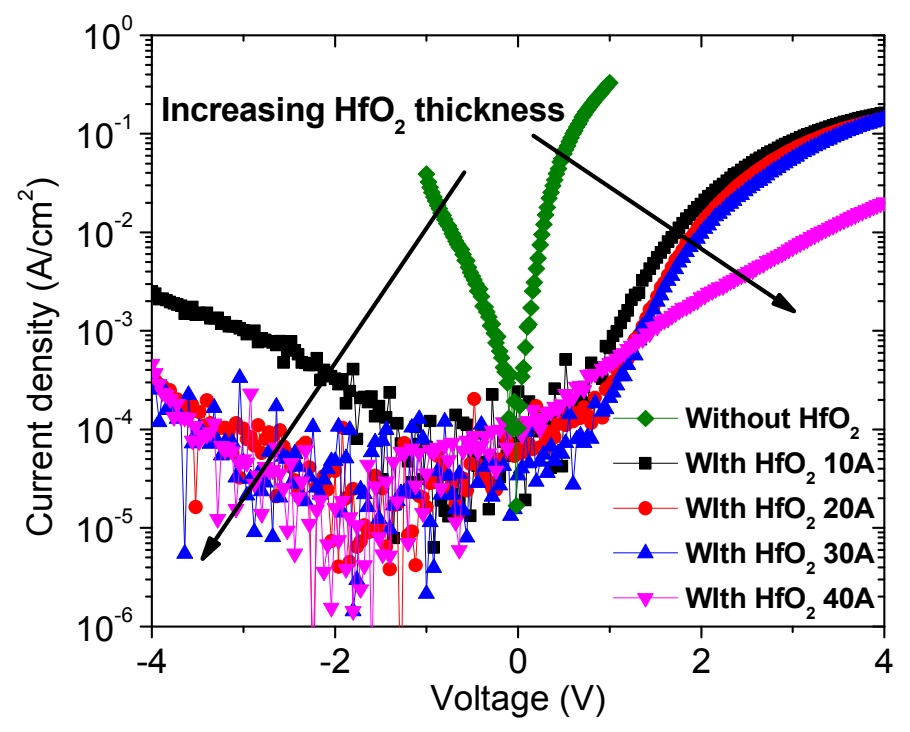

Figure S11. Semi-log I-V curve of $\mathrm{Au} / \mathrm{ZnO}$ Schottky diode $\left(\mathrm{Au} / \mathrm{HfO}_{2} / \mathrm{ZnO} / \mathrm{AZO} / \mathrm{Al}\right)$ with different $\mathrm{HfO}_{2}$ thickness $(0-40 \AA)$ 
Table S1. Calculations from $\log (\mathrm{J})-\mathrm{V}$ plot of $\mathrm{Au} / \mathrm{HfO}_{2} / \mathrm{ZnO}$ MIS Schottky diodes

\begin{tabular}{l|lccc}
\hline \multicolumn{5}{c}{ Schottky diode of Au/HfO2/ZnO/AZO/Al } \\
\hline $\begin{array}{l}\text { Thickness of } \mathrm{HfO}_{2} \\
(\AA)\end{array}$ & $\begin{array}{c}\text { Ideality factor } \\
n\end{array}$ & $\begin{array}{c}\text { Dark Saturation Current } \\
J_{0}\left(\mathrm{~A} / \mathrm{cm}^{2}\right)\end{array}$ & $\begin{array}{c}\text { Barrier Height } \\
\phi_{b}(\mathrm{eV})\end{array}$ \\
\hline \hline 0 & 1.9 & $1.2 \times 10^{-4}$ & 0.61 & \\
10 & 9.1 & $1.1 \times 10^{-5}$ & 0.68 & \\
20 & 8.8 & $3.3 \times 10^{-6}$ & 0.72 & \\
30 & 8.9 & $2.7 \times 10^{-6}$ & 0.72 & \\
40 & 20.8 & $7.6 \times 10^{-5}$ & 0.64 & \\
\hline
\end{tabular}

\section{External quantum efficiency with amplification}

The amplified external quantum efficiency (EQE) of plasmon FET is shown in Figure S12. This data is obtained using data in Figure 3a in the manuscript. This shows that the plasmon FET has over $600 \%$ of amplified EQE (number of output carrier/number of input photon) under $20 \mathrm{~V}$ gate voltage bias.

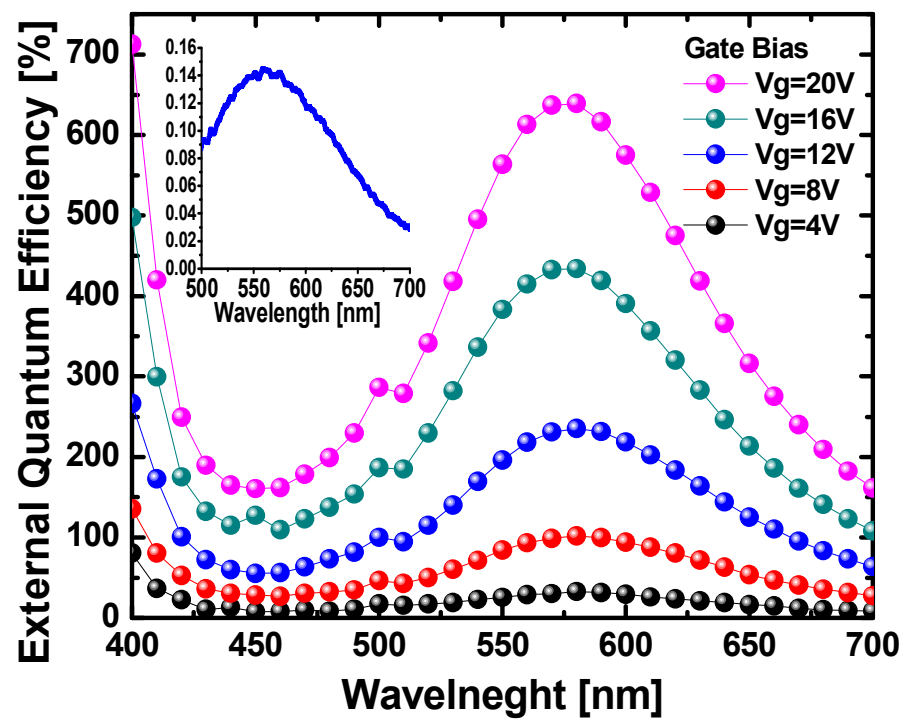

Figure S12. The amplified external quantum efficiency (EQE) of the plasmon FET as a function of gate voltage bias

\section{References}


1. Leenheer, A. J.; Narang, P.; Lewis, N. S.; Atwater, H. A. J. Appl. Phys. 2014, 115, (13), 134301.

2. Liu, K.; Sakurai, M.; Liao, M.; Aono, M. J. Phys. Chem. C 2010, 114, (46), 19835-19839.

3. Razavi, B., Design of Analog CMOS Integrated Circuits. McGraw-Hill: 2001.

4. He, L.; Chen, F. ECS Solid State Lett. 2014, 3, (4), 41-44.

5. Haynes, W. M., CRC handbook of chemistry and physics. CRC Press: 2014. 